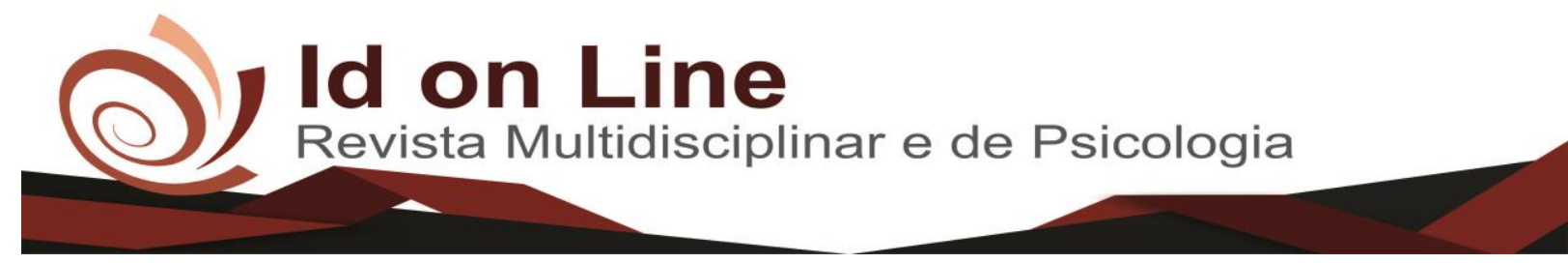

Artigo de Revisão

\title{
Efeito da Terapia Manual em Pacientes com Lombalgia: Uma Revisão Integrativa
}

\author{
Dayana Sales Pereira ${ }^{1}$; Virgílio Santana Junior ${ }^{2}$
}

Resumo: A terapia manual em pacientes com lombalgia é uma técnica muito aplicada para melhorar a flexibilidade lombar de pessoas com e sem dor nesta região. Ela é caracterizada como um dos problemas de saúde mais frequentes na população geral. Metodologia: Trata-se de uma revisão integrativa da literatura científica sobre o efeito da terapia manual em pacientes com lombalgia. As pesquisas foram realizadas a partir das bases de dados SciELO, LILACS e PEDro, no período de março a maio de 2018. Foram selecionados cinco artigos publicados entre 2005 e 2017 que tratavam da temática. Objetivo: Esse estudo teve como objetivo compilar informação a respeito do efeito da terapia manual em pacientes com lombalgia. Resultados: $\mathrm{O}$ trabalho proporcionou incrementar o conhecimento a respeito do tratamento da lombalgia, demonstrando que a técnica é de grande relevância e tem eficácia comprovada na diminuição da dor e, consequentemente, na evolução da capacidade funcional dos pacientes. Conclusão: O presente trabalho demostrou que a técnica de terapia manual é efetiva no tratamento de pessoas com diagnóstico de lombalgia, aumentando a capacidade funcional das mesmas.

Palavras-Chave: lombalgia, terapia manual, fisioterapia.

\section{Effect of Manual Therapy in Low Back Pain Patients: An Integrative Review}

\begin{abstract}
The manual therapy in the low back pain patients is a widely used technique to recover the lumbar flexibility in people with or without pain in this location. It is characterized as one of the most common health problems in the general population. Methodology: It is a scientific literature integrative review on the effect of the manual therapy in low back pain patients. The search was carried out in SciELO, LILACAS and PEDro database, between March and May 2018. Five articles related to the thematic, published from 2005 to 2017, were selected. Objective: This research aimed to compile information related to the effect of manual therapy in low back pain patients. Results: It provided a step up in the knowledge concerned to the low back pain treatment, showing that technique holds great relevance and has proved efficacy in the pain level decrease, and consequently, in the patients' functional capability evolution. Conclusion: This study demonstrated that the manual therapy is effective in the low back pain patients' treatment, and increase their functional capability.
\end{abstract}

Keywords: low back pain, manual therapy, physiotherapy.

\footnotetext{
${ }^{1}$ Graduação em Fisioterapia pela Faculdade Independente do Nordeste. day_sallys@ @hotmail.com

${ }^{2}$ Fisioterapeuta. Especialista em Terapia Manual e Postural pelo CESUMAR (Centro Universitário de Maringá).
} 


\section{Introdução}

A Lombalgia é um sintoma presente em alterações musculoesqueléticas da região lombar que gera incapacidade funcional, estando associada a elevado índice de ausência no trabalho e altos custos para a saúde relacionadas a ela, afetando 70 a $80 \%$ dos cidadãos. Tornase crônica em 10 a $15 \%$ dos indivíduos, impossibilitando a realização das suas atividades laborais, sociais e familiares. No Brasil, é umas das principais causas de fornecimento de auxílio-doença das afecções da coluna e aposentadoria por invalidez. (MENDONÇA; ANDRADE,2016).

Devido uma maior mobilidade de L4-L5 e L5-S1, esses segmentos são os mais comprometidos, pois sustentam o peso do corpo quando comparado ao restante da coluna lombar. O quadro comum de hérnia de disco é a dor, a princípio aguda na região da coluna lombar, tendo uma irradiação em direção à fossa poplítea até o dorso do pé. Além do quadro doloroso, o paciente pode relatar formigamento com déficit de força muscular no membro afetado, levando ao quadro conhecido de lombociatalgia, uma vez que a dor faz o percurso do nervo ciático (MONNERAT et al., 2012).

Cerca de 10 a $15 \%$ da população brasileira com a sintomatologia convive com algum grau de incapacidade na realização de suas atividades laborais, sociais e familiares, podendo ser desenvolvida por uma alteração do disco vertebral, por exemplo, uma hérnia de disco lombar ou alterações musculoesqueléticas. No Brasil, as disfunções associadas à hérnia de disco na coluna cervical, torácica e lombar são as principais causas de fornecimento de auxílio-doença e afastamento dos trabalhos e aposentadoria precoce por invalidez. Na procura pelo atendimento de fisioterapia, nos casos da hérnia de disco cervical, as técnicas que ajudam na melhora do quadro desses pacientes são a tração manual e terapia manual. (BIANCHL et al., 2016).

Entre os vários procedimentos de fisioterapia, um dos mais utilizados é a terapia manual, que é uma das técnicas mais apropriadas para a reabilitação nos casos das dores lombares. Ela tem como maior objetivo promover o alívio das dores e, por consequência, da função biomecânica dos tecidos. Como exemplo tem-se a manipulação vertebral e as mobilizações articulares. (BOSCHI; LIMA, 2012). 
Segundo Souza et al (2011) a terapia manual irá agir promovendo a redução da dor e melhora na amplitude de movimento. A terapia é uma modalidade organizada por vários mecanismos que dispõem os recursos terapêuticos nas sustentações músculos-esqueléticos, com métodos que vão incluir manipulação, mobilização passiva e mobilização neuromuscular.

Dentre as técnicas da terapia manual, está a tração manual, a qual é aplicada nos tratamentos conservadores para a melhora de diversos sintomas, incluindo assim a movimentação vertebral, onde a realização dessa tração vai auxiliar na separação do forame intervertebral, melhorando o ângulo para a realização da tração manual. Pode ser realizada com o paciente em posição de decúbito dorsal. A tração faz parte do gerenciamento de fisioterapia mecânica, os tipos de tração e parâmetros relacionados à sua aplicabilidade que ainda precisam ser explorados. (LAVADO et al., 2011).

Diante de tal discussão, verificou-se a importância de realizar uma revisão integrativa abordando o efeito da terapia manual em pacientes com lombalgia, uma vez que essa patologia gera grande impacto na saúde e qualidade de vida dos indivíduos, o que demonstra a relevância desse trabalho.

Ela pode contribuir no direcionamento da reabilitação de pacientes no que cabe à área da fisioterapia, buscando também a prevenção de mais agravos decorrentes dessa lombalgia. Nesse contexto, o objetivo da pesquisa foi compilar dados referentes aos efeitos da terapia manual em pacientes com lombalgia.

\section{Metodologia}

Trata-se de uma revisão integrativa da literatura, de caráter descritivo, exploratório, sem metanálise, que buscou conhecer o efeito da terapia manual em indivíduos que apresentam lombalgia. Este tipo de pesquisa tem uma proposta de reunir e fornecer conhecimento sobre um definido tema, assim colaborando para o entendimento aprofundado acerca do mesmo. Segundo Sampaio (2007), toda pesquisa integrativa passa por uma investigação das fontes de dados na literatura em relação a determinado assunto. Este conteúdo torna se disponível através de uma síntese de indícios que, através da realização desses métodos serão esclarecidos, a partir da avaliação minuciosa do apanhado de informações escolhidas. A busca na literatura científica foi realizada a partir das bases de dados SciELO, LILACS e PEDro, no período de marco a 
maio de 2018. Os relatores utilizados foram "lombalgia", "fisioterapia" e "terapia manual". Estes foram pesquisados separadamente.

Foram selecionados 49 artigos para produção da amostra, 5 se encaixaram nos critérios de inclusão e foram considerados adequados pois abordavam o efeito da terapia manual em pacientes com lombalgia como foi a temática determinada. Estes foram trabalhos originais publicados entre 2005 e 2017. Foram excluídos da coleta, artigos científicos cujas versões completas não se encontravam disponíveis, artigos que abordavam cirurgias ou que que não abordassem a temática.

Para a realização da tabulação dos dados após a coleta, foi desenvolvida uma tabela de resultados que conta com a identificação dos seguintes tópicos: título, autores, ano de publicação, orientação metodológica, objetivos, população estudada e resultados obtidos.

\section{Resultados}

Tabela 1. Efeito da terapia manual em pacientes com lombalgia.

\begin{tabular}{|c|c|c|c|c|c|}
\hline Título & Autor/Ano & $\begin{array}{l}\text { Orientação } \\
\text { Metodológica }\end{array}$ & Objetivo & Participantes & $\begin{array}{l}\text { Principais } \\
\text { resultados }\end{array}$ \\
\hline $\begin{array}{c}\text { Efeitos da terapia manual } \\
\text { de Maitland em pacientes } \\
\text { com lombalgia crônica. }\end{array}$ & $\begin{array}{l}\text { NAVEGA;TA } \\
\text { MBASCIA } \\
2011 .\end{array}$ & Artigo Original & $\begin{array}{l}\text { Avaliar os efeitos do } \\
\text { tratamento } \\
\text { fisioterapêutico } \\
\text { manipulativo de } \\
\text { Maitland no quadro } \\
\text { álgico, flexibilidade, } \\
\text { capacidade } \\
\text { funcional e } \\
\text { qualidade de vida de } \\
\text { paciente } \\
\text { com lombalgia } \\
\text { crônica. }\end{array}$ & $\begin{array}{l}\text { Os dados foram } \\
\text { obtidos de } \\
\text { pacientes de } \\
\text { Marília, na } \\
\text { faixa etária de } 23 \\
\text { a } 68 \text { anos, de } \\
\text { ambos os sexos, } \\
\text { com } \\
\text { relato de dor } \\
\text { lombar crônica } \\
\text { (queixa por } \\
\text { período maior } \\
\text { que } 12 \text { semanas). }\end{array}$ & $\begin{array}{l}\text { Houve melhora } \\
\text { significativa na } \\
\text { intensidade de dor, } \\
\text { incapacidade, } \\
\text { flexibilidade } \\
\text { e qualidade de vida. }\end{array}$ \\
\hline $\begin{array}{l}\text { Terapia manual em } \\
\text { pacientes portadores } \\
\text { de hérnia discal lombar: } \\
\text { revisão sistemática }\end{array}$ & $\begin{array}{l}\text { LOIOLA et } \\
\text { al,2017 }\end{array}$ & $\begin{array}{l}\text { Estudo de } \\
\text { revisão } \\
\text { sistemática, } \\
\text { transversal e } \\
\text { com estratégia } \\
\text { de análise } \\
\text { quantitativa }\end{array}$ & $\begin{array}{c}\text { Analisar nas bases } \\
\text { de dados as técnicas } \\
\text { de Terapia Manual } \\
\text { utilizadas em } \\
\text { pacientes portadores } \\
\text { de hérnia discal } \\
\text { lombar }\end{array}$ & $\begin{array}{l}\text { Foram incluídos } \\
12 \text { artigos no } \\
\text { período de } 2001 \\
\text { a } 2013 \text { onde } \\
66,4 \%(\mathrm{n}=8) \\
\text { relataram } \\
\text { a terapia manual } \\
\text { como eficaz, } \\
\text { melhorando } \\
\text { alguns } \\
\text { parâmetros nos } \\
\text { pacientes e33,2\% } \\
(\mathrm{n}=4) \text { não }\end{array}$ & $\begin{array}{c}\text { Proporcionou } \\
\text { melhora no quadro } \\
\text { álgico, na } \\
\text { funcionalidade, na } \\
\text { flexibilidade, no } \\
\text { ganho de força e na } \\
\text { mobilidade articular } \\
\text { permitindo que os } \\
\text { pacientes } \\
\text { conseguissem voltar } \\
\text { a realizar as } \\
\text { atividades de vida } \\
\text { diária. }\end{array}$ \\
\hline
\end{tabular}




\begin{tabular}{|c|c|c|c|c|c|}
\hline & & & & $\begin{array}{l}\text { demonstraram } \\
\text { resultados após } \\
\text { alguns exercícios } \\
\text { e alongamentos }\end{array}$ & \\
\hline $\begin{array}{l}\text { Análise da mobilidade } \\
\text { lombar e influência da } \\
\text { terapia manual e } \\
\text { cinesioterapia na } \\
\text { lombalgia }\end{array}$ & $\begin{array}{l}\text { BRIGANÓ; } \\
\text { MACEDO, } \\
2005\end{array}$ & $\begin{array}{l}\text { Este estudo } \\
\text { caracterizou-se } \\
\text { como um ensaio } \\
\text { clínico não } \\
\text { aleatório. }\end{array}$ & $\begin{array}{c}\text { Objetivo foi } \\
\text { comparar os efeitos } \\
\text { da terapia manual e } \\
\text { cinesioterapia em } \\
\text { pacientes com } \\
\text { lombalgia, } \\
\text { bem como a } \\
\text { mobilidade lombar } \\
\text { de indivíduos com e } \\
\text { sem dor nesta região }\end{array}$ & $\begin{array}{l}\text { Utilizou-se uma } \\
\text { amostra de } \\
\text { conveniência } \\
\text { composta por } 25 \\
\text { indivíduos } \\
\text { com idade entre } \\
18 \text { - } 65 \text { anos, } \\
\text { diagnóstico } \\
\text { clínico de } \\
\text { lombalgia } \\
\text { crônica; foram } \\
\text { excluídos os } \\
\text { pacientes com } \\
\text { cirurgias } \\
\text { lombares, } \\
\text { doenças } \\
\text { reumáticas, } \\
\text { fraturas e } \\
\text { submetidos a } \\
\text { outro tratamento } \\
\text { clínico. }\end{array}$ & $\begin{array}{c}\text { Foi encontrada } \\
\text { diferença } \\
\text { estatisticamente } \\
\text { significante na } \\
\text { comparação da EVA } \\
\text { antes e após o } \\
\text { tratamento } \\
\text { fisioterápico } \\
\text { ( } \mathrm{p}<0,05) \text { e } \\
\text { também para a } \\
\text { mobilidade da } \\
\text { coluna lombar em } \\
\text { indivíduos } \\
\text { sintomáticos e } \\
\text { assintomáticos }\end{array}$ \\
\hline $\begin{array}{l}\text { Terapias Manuais na } \\
\text { Lombalgia: Revisão da } \\
\text { literatura }\end{array}$ & $\begin{array}{c}\text { BARROS, } \\
2013\end{array}$ & $\begin{array}{l}\text { Estudo de } \\
\text { Revisão da } \\
\text { literatura }\end{array}$ & $\begin{array}{l}\text { Tendo como objetivo } \\
\text { revisar a literatura } \\
\text { dos últimos dez anos } \\
\text { para analisar a } \\
\text { eficácia das terapias } \\
\text { manuais na } \\
\text { Lombalgia }\end{array}$ & $\begin{array}{c}\text { Os efeitos da } \\
\text { terapia manual e } \\
\text { cinesioterapia em } \\
\text { pacientes com } \\
\text { lombalgia, bem } \\
\text { como a } \\
\text { mobilidade } \\
\text { lombar de } \\
\text { indivíduos com e } \\
\text { sem dor nesta } \\
\text { região. Para isso } \\
\text { foram utilizadas } \\
\text { amostras de } \\
\text { conveniência, } \\
\text { compostas por } 25 \\
\text { pacientes com o } \\
\text { diagnóstico de } \\
\text { lombalgia } \\
\text { crônica e um } \\
\text { grupo controle } \\
\text { com } 25 \\
\text { indivíduos } \\
\text { assintomáticos, } \\
\text { com a mesma } \\
\text { idade e gênero } \\
\text { dos indivíduos } \\
\text { com lombalgia }\end{array}$ & $\begin{array}{c}\text { Os resultados } \\
\text { obtidos na } \\
\text { mostraram eficácia } \\
\text { das terapias manuais } \\
\text { na eliminação da dor } \\
\text { e na melhora da } \\
\text { qualidade de vida de } \\
\text { pessoas com } \\
\text { lombalgia/dor } \\
\text { lombar, os resultado } \\
\text { foram positivo na } \\
\text { sintomatologia } \\
\text { dolorosa e promoveu } \\
\text { diminuição das } \\
\text { incapacidades. }\end{array}$ \\
\hline $\begin{array}{l}\text { Aplicação da Terapia de } \\
\text { Liberação Posicional } \\
\text { em Paciente com } \\
\text { Lombalgia Crônica de } \\
\text { Origem Mecânica }\end{array}$ & $\begin{array}{c}\text { MOURA; } \\
\text { SILVA, } 2006 .\end{array}$ & Estudo de Caso & $\begin{array}{l}\text { O objetivo desse } \\
\text { estudo foi avaliar e } \\
\text { tratar um paciente } \\
\text { lombálgico através } \\
\text { da Terapia de } \\
\text { Liberação } \\
\text { Posicional. }\end{array}$ & $\begin{array}{c}\text { O presente } \\
\text { trabalho trata-se } \\
\text { de } \\
\text { um estudo de } \\
\text { caso clínico de } \\
\text { caráter } \\
\text { prospectivo e } \\
\text { intervenciona de }\end{array}$ & $\begin{array}{c}\text { Os resultados } \\
\text { mostraram que } \\
\text { esta técnica trouxe } \\
\text { de fato benefícios } \\
\text { para a paciente. } \\
\text { Foi observado } \\
\text { que entre a } \\
\text { dor e restrição }\end{array}$ \\
\hline
\end{tabular}




\begin{tabular}{|l|l|l|c|c|}
\hline & & & $\begin{array}{c}\text { uma paciente do } \\
\text { sexo feminino, } \\
47 \\
\text { anos, casada, } \\
\text { durante a primeira } \\
\text { avaliaçãa, a maioria } \\
\text { retornou } \\
\text { ensora do } \\
\text { ao seu tônus normal } \\
\text { após a } \\
\text { ensino } \\
\text { fundamental e } \\
\text { médilização dessa }\end{array}$ \\
\hline
\end{tabular}

Fonte: SciELO, LILACS e PEDro.

\section{Discussão}

Esta revisão integrativa apresentou que há confirmação de evidências cientificas indicando que a técnica de terapia manual é eficiente, uma vez que alcançou uma evolução no tratamento da lombalgia e da capacidade funcional dos indivíduos, melhorando a eficácia neuromuscular e prevenindo lesões.

Navega et al, (2011) destaca que a técnica de terapia manual promoveu significativa eficácia no tratamento conservador na dor lombar, tendo benefícios em cerca de $70 \%$ dos indivíduos, no período de quatro a cinco semanas de intervenção.

Já no estudo feito por Loila et al, (2017) mostra os principais benefícios de exercícios já bem conhecidos como sendo adequados à sintomatologia e incentivam os pacientes a executarem atividades físicas que frequentemente é bastante eficiente para quem apresenta essa patologia. Ele também relata que a hérnia de disco é uma desarticulação músculo-esqueletica, causador pela lombociatalgia.

Briganó et al (2005) e Barros et al (2013) apresentam que a terapia manual tem uma atuação significativa no desenvolvimento da lombalgia e da flexibilidade da coluna lombar, quando são utilizadas técnicas que promovem o relaxamento muscular, melhorando cada vez mais o quadro álgico dos pacientes.

Moura; Silva (2006) mostraram que a técnica de terapia manual, trouxe benefícios importantes referentes à lombalgia como o planejamento em relação à dor e ao tônus dos pacientes que apresentam essa patologia.

Em outra concepção, Brandão et. al. (2014) colabora que as evidencia sobre a aplicação da técnica de terapia manual de sessões e intervenções inferiores que duas vezes por semana não teve relevância quando realizada em uma pequena amostra de indivíduos com lombalgia. 
Sendo assim, é interessante destacar que realizar uma frequência maior de intervenções vai gerar bons resultados no tratamento conservador da dor lombar.

Os estudos mostram que a técnica de terapia manual tem resultados satisfatórios, demonstrando eficiência, diminuição da dor e inabilidade, nos casos de lombalgias tanto aguda quanto crônica.

\section{Conclusão}

Percebe-se que esta pesquisa conceituou cientificamente que a técnica de terapia manual apresentou ser efetiva no cuidado e no tratamento de pessoas com diagnóstico de lombalgia, aumentando a capacidade funcional destes. Esta técnica manifesta ter resultados significativos, possibilitando a melhora na eficácia funcional e proporcionando uma melhor qualidade de vida a população, evitando assim, que muitos sejam submetidos ao processo cirúrgico.

Com isto, pretende-se contribuir para o conhecimento dos profissionais e acadêmicos no intuito de que eles sejam qualificados a realizar um tratamento eficaz para esses pacientes, levando em conta às necessidades e individualidades de cada um.

O trabalho colabora para o avanço de novos estudos que possam aprofundar os dados encontrados e fortalecer linhas de pesquisas relacionadas ao tratamento das desordens musculoesqueléticas, uma vez que existem poucos estudos que demonstre a eficácia da técnica.

\section{Referências}

ALENCAR, G. G; Comparação do efeito da Facilitação Neuromuscular Proprioceptiva e da Estabilização Segmentar Vertebral na dor lombar e nas dimensões dos músculos multífidos e transverso do abdome em pacientes com hérnia de disco. Dissertação de Mestrado, Universidade Federal de Pernambuco, 2016.

ALFIERI, F. M. et. al.; Comparação da flexibilidade, intensidade da dor e funcionalidade de portadores de hérnia discal submetidos à hidrocinesioterapia versus cinesioterapia clássica. Life Style Journal, p. 45-53, $1^{\circ}$ semestre, São Paulo 2015.

BIAUCHL, A. B. et. al. Estudo comparativo entre os métodos Pilates no solo e Water Pilates na qualidade de vida e dor de pacientes com lombalgia. Revista do Departamento de 
Educação Física e Saúde e do Mestrado em Promoção da Saúde da Universidade de Santa Cruz do Sul / Unisc, v. 17, n. 4, Outubro/Dezembro 2016

BOSEHI,S.E; LIMA,C.D. Efeitos da manipulação torácica na dor e amplitude de movimento da coluna cervical. Revista de Iniciação Cientifica do Unilasalle, v.1.n.1, p.78-91, maio 2012.

KOBILL, A. F. M. Influência da Estabilização Segmentar core na dor e funcionalidade da coluna lombar. Fisioterapia Brasil, v. 18, n. 2, p. 148-153, 2017.

LOVADO, S..et,al.Protocolo de Tratamento para Hérnia de Disco Cervical: Estudo de Caso. Revista Eletrônica Saúde: Pesquisa e Reflexões. V.1,n1,p01-21,2011.

MONNERAT, E. et. al. Efeito da Mobilização Neural na melhora da dor e incapacidade funcional da hérnia de disco lombar subaguda. Fisioterapia Brasil, v. 13, n.1, janeiro/fevereiro de 2012.

MENDONÇA, E. M. T.; ANDRADE, T.M. Método Mckenzie como protocolo de tratamento em hérnia de disco lombar. Revista Interdisciplinar, v. 9, n. 3, p. 130-137, jul. ago. set. 2016.

SAMPAIO R. F.; MANCINI, M. C. Estudos de revisão sistemática: um guia para síntese criteriosa da evidência científica. Departamento de Fisioterapia e de Terapia Ocupacional, Escola de Educação Física, Fisioterapia e Terapia Ocupacional, Universidade Federal de Minas Gerais, Belo Horizonte, MG - Brasil. Rev. Bras. Fisioter. São Carlos, v. 11, n. 1, p. 83-89, jan. / fev. 2007.

SOUZA.et,al. Protocolo de terapia manual no tratamento para Hérnia de Disco: Estudo de Caso. Revista Eletrônica Saúde: Pesquisa e Reflexões. V.1,n1,p01-21,2011.

\section{Como citar este artigo (Formato ABNT):}

PEREIRA, Dayana Sales; SANTANA JUNIOR, Virgílio. Efeito da Terapia Manual em Pacientes com Lombalgia: Uma Revisão Integrativa. Id on Line Revista Multidisciplinar e de Psicologia, 2018, vol.12, n.41, p.31-38. ISSN: 1981-1179.

Recebido: 30.05 .2018

Aceito: 06.06.2018 